\title{
Dynamic Analysis of a Phytoplankton-Fish Model with the Impulsive Feedback Control Depending on the Fish Density and Its Changing Rate
}

Jingli Fu ( $\square$ sqfujingli@163.com )

Zhejiang University of Water Resources and Electric Power

Xiaoyu Hou

Shandong University of Science and Technology

Tonghua Zhang

Swinburne University of Technology

Huidong Cheng

Shandong University of Science and Technology

\section{Research Article}

Keywords: Poincaré map, current growth rate, population density of fish, impulse feedback control, periodic solution.

Posted Date: November 29th, 2021

DOI: https://doi.org/10.21203/rs.3.rs-981564/v1

License: (c) (i) This work is licensed under a Creative Commons Attribution 4.0 International License. Read Full License 


\title{
Dynamic analysis of a phytoplankton-fish model with the impulsive feedback control depending on the fish density and its changing rate
}

\author{
Jingli Fu ${ }^{1}$, Xiaoyu Hou ${ }^{2}$, Tonghua Zhang ${ }^{3}$, Huidong Cheng ${ }^{2 *}$ \\ 1.College of Mechanical and Automotive Engineering, Zhejiang University of water Resources and Electric \\ Power, Hangzhou 310018, China \\ 2.College of Mathematics and System Sciences, Shandong University of Science and Technology \\ Qingdao 266590, Shandong, People's Republic of China \\ 3.Department of Mathematics, Swinburne University of Technology, Australia 3122
}

\begin{abstract}
This paper proposes a comprehensive fishing strategy that takes into consideration the population density of fish and its current growth rate, which provides new ideas for fishing strategies. Firstly, we establish a phytoplankton-fish model with the impulsive feedback control depending on the density and rate of change of the fish. Secondly, the complex phase and impulse sets of this model are divided into three cases, then the Poincaré map for the model is defined, and analyzed the properties of Poincaré map. In addition, the sufficient and necessary conditions for the global asymptotic stability of the order- 1 periodic solution and existence condition of $\operatorname{order}-k(k \geq 2)$ periodic solution are discussed. The action threshold depends on the density and rate of change of the fish, which is reasonable than earlier studies. The analysis method proposed in this paper also plays an important role in the analysis of impulse models with complex phase sets or impulse sets.
\end{abstract}

Keywords: Poincaré map, current growth rate, population density of fish, impulse feedback control, periodic solution.

\section{Introduction}

Numerous plankton models have been developed over the years due to the importance of plankton, which includes both phytoplankton and zooplankton, to marine ecosystems, for example, the interaction model of phytoplankton and zooplankton with time lags proposed by [1] and the predation

\footnotetext{
${ }^{*}$ Corresponding author Huidong Cheng; E-mil:chd900517@sdust.edu.cn(Huidong Cheng), Tel:0532-88032097.
} 
model of phytoplankton and fish with commercial value in [2], where the impact of different fishing levels on fisheries was discussed. Fish is not only a beneficial source of food, but also provides an important economic source. Recent studies [3-9] suggest continuous or over harvesting on a particular species may cause the extinction of that species of fish. This motivates researchers to conduct more in-depth research on fishing, such as how and when to implement capture strategies? Mathematical models are employed and play an important role in this kind of study, in which a basic assumption is that the capture is carried out as soon as the density of the population reaches a given threshold. Then the resulting models can be described by systems of impulsive differential equations [10-16], where the impulse set and phase set in the impulse model are two straight lines due to the assumption. Then theories of impulsive semi-dynamic systems, which is also known as a semi-continuous dynamic system can be applied to investigate the model dynamics, such as Zeng et al. generalised the Poincaré-Bendixon theorem of ordinary differential equations to explore the periodic solution of the model [17]. Jiang et al. obtained the order-1 periodic solutions using the Poincaré map $[18,19]$, and Chen developed the idea of successor functions to study the mathematical models with pulse state feedback control [20]. Due to its importance in applications, in recent years, systems of impulsive differential equations have attracted more and more attention and been applied to different areas from population dynamics to chemical regulator systems [21-28]. Due to the challenges in analysing these models, most of existing models only considered the population size without considering the population growth rate when proposing a control strategy $[29,30]$. However it would be more reasonable if both growth rate and population size are taken into consideration when developing a capture strategy.

The main purpose of this research is twofold: (a) to improve a phytoplankton-fish model by proposing an impulse feedback control that incorporates not only the population density but also the population growth rate; (b) to propose a way for calculating the Poincaré map when the impulse set and phase set are curves instead of straight lines. More precisely, in this paper, we

proposes a comprehensive control measure that takes into consideration the population density of fish and its current growth rate based on the fish density control model in [31]. The rest of paper is arranged as follows: In section 2, we propose a new phytoplankton-fish capture model with a new type of control strategy by taking into account the density of fish growth in addition to the current number of fish. In section 3, we construct the Poincaré maps for the model, and then some properties of Poincaré map are analyzed. Then this is followed by discussing the existence of the order- $k(k \geq 1)$ periodic solution of the model, and the uniqueness and global stability of order-1 periodic solution in section 4 . We then carry out some numerical simulations in section 5 and finally conclude our study in section 6 .

\section{Model establishment}

To formulate our model, we first introduce the following notations: 
$u$ : the population of phytoplankton

$v$ : the population of fish

$r$ : the intrinsic growth rate of phytoplankton

$\alpha$ : the absorption rate of phytoplankton by fish

$\beta$ : the conversion rate of biomass

$d$ : the mortality rate of fish

$H$ : the threshold at which fish are allowed to be caught

$\delta$ : the maximum capture rate

$\gamma:$ the half-saturation constant

$\tau$ : the reduced number of phytoplankton

$\theta$ : the morphology parameters.

Furthermore let $\frac{a u}{b+u}$ represent the number of death fish due to the distribution of phytoplankton toxicants, and all parameters are positive and $0<\delta<1$. Then, Li, Liu and Cheng proposed the following phytoplankton-fish model with the impulsive feedback control [31].

$$
\left\{\begin{array}{l}
\frac{d u}{d t}=(r-\alpha v(t)) u(t), \\
\frac{d v}{d t}=\left(\beta u(t)-d-\frac{a u(t)}{b+u(t)}\right) v(t), \\
u\left(t^{+}\right)=u(t)\left(1-\frac{\delta u(t)}{u(t)+\gamma}\right) \\
v\left(t^{+}\right)=v(t)-\frac{\tau}{1+\theta v(t)},
\end{array}\right\} v=H,
$$

Now it is known that the state pulse capture strategy in (1) has certain shortcomings as it only considers the population size and does not consider the population growth rate. To overcome these shortcomings, we propose a density and rate-dependent feedback control model, in which we will have curves as the pulse set and phase set, which is different from the existing works and brings extra difficulties in analysing the model. More precisely, the control threshold is described by

$$
a_{1} v+b_{1} \frac{d v}{d t}=H
$$

where $a_{1}$ and $b_{1}$ are positive constants, satisfying $a_{1}+b_{1}=1$, and they are the weight coefficients that control the rate of change in fish population and the rate of change in fish population density, respectively. Biologically, equation (2) implies that fish catching is allowed only when the fish population $v$ satisfies $a_{1} v+b_{1} \frac{d v}{d t} \geq H$. Then we reach a model in the following form: 


$$
\left\{\begin{array}{l}
\frac{d u}{d t}=(r-\alpha v(t)) u(t), \\
\frac{d v}{d t}=\left(\beta u(t)-d-\frac{a u(t)}{b+u(t)}\right) v(t), \\
u\left(t^{+}\right)=u(t)\left(1-\frac{\delta u(t)}{u(t)+\gamma}\right), \\
v\left(t^{+}\right)=v(t)-\frac{\tau}{1+\theta v(t)},
\end{array}\right\} a_{1} v+b_{1} \frac{d v}{d t}<H,
$$

which is known as a semi-continuous dynamic system $[32,33]$. Without the feedback control, the system (3) reduces to an ODE system

$$
\left\{\begin{aligned}
\frac{d u}{d t} & =(r-\alpha v(t)) u(t) \\
\frac{d v}{d t} & =\left(\beta u(t)-d-\frac{a u(t)}{b+u(t)}\right) v(t) .
\end{aligned}\right.
$$

A straightforward analysis or by [34] shows system (4) has two equilibrium points: a saddle point, $O(0,0)$ and a stable positive equilibrium $E^{*}\left(u^{*}, v^{*}\right)$, where

$$
v^{*}=\frac{r}{\alpha}, \quad u^{*}=\frac{d+a-\beta b+\sqrt{(d+a-\beta b)^{2}-4 \beta b d}}{2 \beta}
$$

determined by the intersection of the two nullclines

$$
L_{1}: \nu=\frac{r}{\alpha} ; \quad L_{2}: u=\frac{d+a-\beta b+\sqrt{(d+a-\beta b)^{2}-4 \beta b d}}{2 \beta} .
$$

Next we construct the Poincaré map to investigate the dynamics of (3).

\section{Poincaré map of system (3) and its properties}

By using the equation (2) and the second equation in (3), we obtain

$$
v=\frac{H}{a_{1}+b_{1} \beta u-b_{1} d-\frac{a b_{1} u}{b+u}} .
$$

From biological point of view we only consider the properties of the model in region $R_{2}^{+}=$ $\{(u, v), u \geq 0, v \geq 0\}$. To reveal the properties of the Poincaré map, without loss of generality, we assume the initial population size $v_{0}^{+}$of the fish population satisfies

$$
a_{1} v_{0}^{+}+b_{1}\left(d v_{0}^{+} / d t\right)<H
$$

and the initial point $\left(u_{0}^{+}, v_{0}^{+}\right)$satisfies

$$
v=\frac{H}{a_{1}+b_{1} \beta \sigma(u)-b_{1} d-\frac{a b_{1} \sigma(u)}{b+\sigma(u)}} .
$$

Then, in order to define the impulse set and phase set of the model we introduce the following sets

$$
\begin{aligned}
& \Sigma_{N}=\left\{(u, v), u \geq 0, v \geq 0, v=\frac{H}{a_{1}+b_{1} \beta \sigma(u)-b_{1} d-\frac{a b_{1} \sigma(u)}{b+\sigma(u)}}\right\}, \\
& \Sigma_{M}=\left\{(u, v), u \geq 0, v \geq 0, v=\frac{H}{a_{1}+b_{1} \beta u-b_{1} d-\frac{a b_{1} u}{b+u}}\right\},
\end{aligned}
$$


where

$$
\sigma(u)=\frac{-\varepsilon+u+\sqrt{-4 \varepsilon \delta u+\varepsilon^{2}+2 \varepsilon u+u^{2}}}{2(1-\delta)} .
$$

We further assume $\Sigma_{N}$ and $\Sigma_{M}$ intersect with $L_{2}$ at $A^{+}$and $A^{-}$respectively, then assume $\Sigma_{N}$ and $\Sigma_{M}$ intersect with $x$ at $B^{1}\left(u^{1}, 0\right)$ and $B^{2}\left(u^{2}, 0\right)$, respectively. Then depending on the locations of $\Sigma_{N}$ and $v^{*}$, namely the positive equilibrium point we can define the impulse set and phase set as follows.

\subsection{Cases I: $v^{*} \geq \frac{H}{a_{1}+b_{1} \beta u^{*}-b_{1} d-\frac{a b_{1} u^{*}}{b+u^{*}}}$.}

In this case, $E^{*}$ is above the impulse set $\Sigma_{M}$ (see Fig. 1(a)). Thus, there exists a point $T$ on the $\Sigma_{N}$ at which the trajectory of (3) is tangent to the $\Sigma_{N}$. Denote the intersection between the trajectory and $\Sigma_{M}$ by $Q_{1}$. Then we define the impulse set by

$$
M_{1}=\left\{(u, v) \mid v=\frac{H}{a_{1}+b_{1} \beta u-b_{1} d-\frac{a b_{1} u}{b+u}}, u \geq u_{Q_{1}}\right\}
$$

and the phase set by

$$
N_{1}=\left\{(u, v) \mid v=\frac{H}{a_{1}+b_{1} \beta \sigma(u)-b_{1} d-\frac{a b_{1} \sigma(u)}{b+\sigma(u)}}, \quad u \geq u_{Q_{1}}\left(1-\frac{\delta u_{Q_{1}}}{u_{Q_{1}}+\gamma}\right)\right\} .
$$

\subsection{Cases II: $v^{*}<\frac{H}{a_{1}+b_{1} \beta u^{*}-b_{1} d-\frac{a b_{1} u^{*}}{b+u^{*}}}$ and $v_{W^{-}}>\frac{H}{a_{1}+b_{1} \beta \sigma\left(u^{*}\right)-b_{1} d-\frac{a b_{1} \sigma\left(u^{*}\right)}{b+\sigma\left(u^{*}\right)}}$.}

In this case, $E^{*}$ is below the impulse set $\Sigma_{M}$ (see Fig. 1(b)). We first assume that $\Gamma_{M}$ is the trajectory passing through $M$ in the system. If there is a point $W$ on the $\Sigma_{M}$ so that $\Gamma_{W}$ is just tangent to the $\Sigma_{M}$, when $v^{*}<\frac{H}{a_{1}+b_{1} \beta u^{*}-b_{1} d-\frac{a b_{1} u^{*}}{b+u^{*}}}$ and $v_{W^{-}}>\frac{H}{a_{1}+b_{1} \beta \sigma\left(u^{*}\right)-b_{1} d-\frac{a b_{1} \sigma\left(u^{*}\right)}{b+\sigma\left(u^{*}\right)}}$, there must be a point $T$ on the $\Sigma_{N}$ so that $\Gamma_{T}$ is just tangent to the $\Sigma_{N}$ and $\Gamma_{T}$ tangent to the $\Sigma_{M}$ at the point $Q_{2}$. Then, in this case, we define the impulse set by

$$
M_{2}=\left\{(u, v) \mid v=\frac{H}{a_{1}+b_{1} \beta u-b_{1} d-\frac{a b_{1} u}{b+u}}, u \geq u_{Q_{2}}\right\},
$$

and the corresponding phase set by

$$
N_{2}=\left\{(u, v) \mid v=\frac{H}{a_{1}+b_{1} \beta \sigma(u)-b_{1} d-\frac{a b_{1} \sigma(u)}{b+\sigma(u)}}, \quad u \geq u_{Q_{2}}\left(1-\frac{\delta u_{Q_{2}}}{u_{Q_{2}}+\gamma}\right)\right\} .
$$



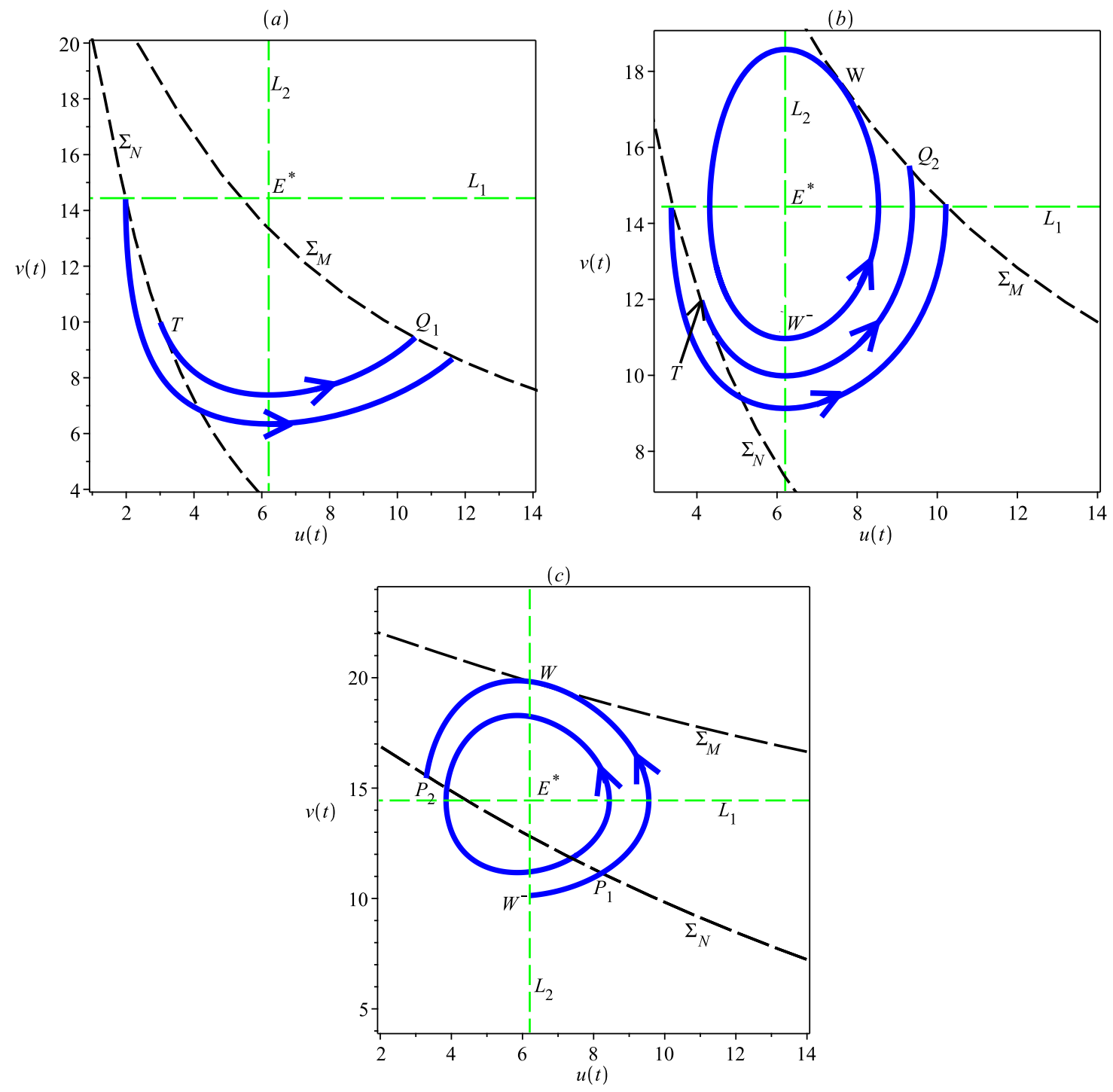

Figure 1: The phase set and impulsive set for Cases I, II and III, respectively. 


\subsection{Cases III: $v_{W^{-}}<\frac{H}{a_{1}+b_{1} \beta \sigma\left(u^{*}\right)-b_{1} d-\frac{a b_{1} \sigma\left(u^{*}\right)}{b+\sigma\left(u^{*}\right)}}$.}

In this case, $E^{*}$ is below the impulse set $\Sigma_{M}$, as shown in Fig. 1(c). Thus, there is a trajectory denoted by $\Gamma_{W^{-}}$that is tangent to $\Sigma_{M}$ at $W$ and intersects the $\Sigma_{N}$ at point $P_{1}$ and point $P_{2}$, respectively. Thus we define the impulse set as

$$
M_{3}=\left\{(u, v) \mid v=\frac{H}{a_{1}+b_{1} \beta u-b_{1} d-\frac{a b_{1} u}{b+u}}, u \geq u_{W}\right\},
$$

and the phase set as

$$
N_{3}=\left\{(u, v) \mid v=\frac{H}{a_{1}+b_{1} \beta \sigma\left(u^{*}\right)-b_{1} d-\frac{a b_{1} \sigma\left(u^{*}\right)}{b+\sigma\left(u^{*}\right)}}, \quad u \in\left(0, u_{P_{2}}\right] \cup\left[u_{P_{1}},+\infty\right)\right\} .
$$

Based on the above defined impulse and phase sets, we are now ready to construct the Poincaré map. To this end, let

$$
A_{k}^{+}=\left(u_{k}^{+}, v_{k}^{+}\right)=\left(u_{k}^{+}, \frac{H}{a_{1}+b_{1} \beta \sigma\left(u_{k}^{+}\right)-b_{1} d-\frac{a b_{1} \sigma\left(u_{k}^{+}\right)}{b+\sigma\left(u_{k}^{+}\right)}}\right) \in \Sigma_{N},
$$

where $0<u_{k}^{+}<+\infty$, and define the trajectory

$$
\pi\left(t, t_{0}, A_{k}^{+}\right) \triangleq\left(u\left(t, t_{0}, A_{k}^{+}\right), v\left(t, t_{0}, A_{k}^{+}\right)\right)
$$

passing through $A_{k}^{+}$that will reach the $\Sigma_{M}$ at point $A_{k+1}\left(u_{k+1}, v_{k+1}\right)$ at time $t_{1}$, where

$$
v_{k+1}=v\left(t_{1}, t_{0},\left(u_{k}^{+}, v_{k}^{+}\right)\right)=\frac{H}{a_{1}+b_{1} \beta u_{k+1}-b_{1} d-\frac{a b_{1} u_{k+1}}{b+u_{k+1}}} .
$$

Then, there is

$$
\begin{array}{r}
u_{k+1}^{+}=u\left(t_{1}, t_{0},\left(u_{k}^{+}, \frac{H}{a_{1}+b_{1} \beta \sigma\left(u_{k}^{+}\right)-b_{1} d-\frac{a b_{1} \sigma\left(u_{k}^{+}\right)}{b+\sigma\left(u_{k}^{+}\right)}}\right)\right) \\
\triangleq u\left(u_{k}^{+}, \frac{H}{a_{1}+b_{1} \beta \sigma\left(u_{k}^{+}\right)-b_{1} d-\frac{a b_{1} \sigma\left(u_{k}^{+}\right)}{b+\sigma\left(u_{k}^{+}\right)}}\right) \triangleq P\left(u_{k}^{+}\right) .
\end{array}
$$

It means that $u_{k+1}^{+}$is determined by $u_{k}^{+}$. Since point $A_{k+1}$ is on the impulse set, $A_{k+1}$ jump to point

$$
A_{k+1}^{+}\left(u_{k+1}^{+}, \frac{H}{a_{1}+b_{1} \beta \sigma\left(u_{k+1}^{+}\right)-b_{1} d-\frac{a b_{1} \sigma\left(u_{k+1}^{+}\right)}{b+\sigma\left(u_{k+1}^{+}\right)}}\right),
$$

where

$$
u_{k+1}^{+}=\left(1-\frac{\delta u_{k+1}}{u_{k+1}+\gamma}\right) u_{k+1}=\left(1-\frac{\delta P\left(u_{k}^{+}\right)}{P\left(u_{k}^{+}\right)+\gamma}\right) P\left(u_{k}^{+}\right) \triangleq G_{m}\left(u_{k}^{+}\right) .
$$


From model (3), we obtain

$$
\left\{\begin{array}{l}
\frac{d u}{d v}=\frac{(r-\alpha v) u}{\left(\beta u-d-\frac{a u}{b+u}\right) v} \triangleq w(u, v), \\
u\left(\frac{H}{a_{1}+b_{1} \beta \sigma\left(u_{k}^{+}\right)-b_{1} d-\frac{a b_{1} \sigma\left(u_{k}^{+}\right)}{b+\sigma\left(u_{k}^{+}\right)}}\right)=u_{0}^{+} .
\end{array}\right.
$$

Let $v_{0}^{+}=\frac{H}{a_{1}+b_{1} \beta \sigma\left(u_{k}^{+}\right)-b_{1} d-\frac{a b_{1} \sigma\left(u_{k}^{+}\right)}{b+\sigma\left(u_{k}^{+}\right)}}, u_{0}^{+}=S$, then we obtain that $\left(u_{0}^{+}, v_{0}^{+}\right)$is in $\Sigma_{N}$.

We define

$$
u(v)=u\left(\frac{H}{a_{1}+b_{1} \beta \sigma\left(u_{k}^{+}\right)-b_{1} d-\frac{a b_{1} \sigma\left(u_{k}^{+}\right)}{b+\sigma\left(u_{k}^{+}\right)}}, v\right) \triangleq u(S, v) .
$$

Then, according to the model (6),

$$
u(S, v)=S+\int_{\frac{H}{a_{1}+b_{1} \beta \sigma\left(u_{k}^{+}\right)-b_{1} d-\frac{a b_{1} \sigma\left(u_{k}^{+}\right)}{b+\sigma\left(u_{k}^{+}\right)}}}^{v} \omega(u(S, s), s) d s .
$$

From (5) and (7), the Poincaré map expression of system (2) is

$$
G_{m}(S)=\left(\begin{array}{c}
\delta \mu\left(\frac{H}{a_{1}+b_{1} \beta \sigma\left(u_{k}^{+}\right)-b_{1} d-\frac{a b_{1} \sigma\left(u_{k}^{+}\right)}{b+\sigma\left(u_{k}^{+}\right)}}, S\right) \\
\mu\left(\frac{H}{a_{1}+b_{1} \beta \sigma\left(u_{k}^{+}\right)-b_{1} d-\frac{a b_{1} \sigma\left(u_{k}^{+}\right)}{b+\sigma\left(u_{k}^{+}\right)}}, S\right)+\gamma
\end{array}\right) \mu\left(\frac{H}{a_{1}+b_{1} \beta \sigma\left(u_{k}^{+}\right)-b_{1} d-\frac{a b_{1} \sigma\left(u_{k}^{+}\right)}{b+\sigma\left(u_{k}^{+}\right)}}, S\right) .
$$

Then we can show that it has the following properties summarised in Theorem 3.1.

Theorem 3.1. If $\frac{H}{a_{1}+b_{1} \beta u^{*}-b_{1} d-\frac{a b_{1} u^{*}}{b+u^{*}}}<v^{*}$, then the trajectory $\Gamma_{T}$ intersects with $\Sigma_{M}$ at point $Q_{1}\left(u_{Q_{1}}, v_{Q_{1}}\right)$, and furthermore we have

(i) The domain of $G_{m}(S)$ is $\left(0, u^{1}\right), G_{m}(S)$ is monotonically decreasing on $\left(0, u_{T}\right]$, and monotonically increasing on $\left(u_{T}, u^{1}\right)$.

(ii) $G_{m}(S)$ is continuously differentiable on $\left(0, u^{1}\right)$.

(iii) When $G_{m}\left(u_{T}\right)>u_{T}$, it has a unique fixed point on $\left(u_{T},+\infty\right)\left(\right.$ see Fig. 2(b)). When $G_{m}\left(u_{T}\right)<$ $u_{T}$, it has a unique fixed point on $\left(0, u_{T}\right)$ (see Fig. 2(a)). When $G_{m}\left(u_{T}\right)=u_{T}, u_{T}$ is the fixed point.

Proof. (i) Notice $E^{*}\left(u^{*}, v^{*}\right)$ is a linear center and $\frac{H}{a_{1}+b_{1} \beta u^{*}-b_{1} d-\frac{a b_{1} u^{*}}{b+u^{*}}}<v^{*}$. Taking any point $A_{k}^{+}\left(u_{k}^{+}, v_{k}^{+}\right)$on $\Sigma_{N}$, the trajectory $\Gamma_{A_{k}^{+}}$will reach the $M_{1}$. So the domain of $G_{m}(S)$ is $\left(0, u^{1}\right)$. 

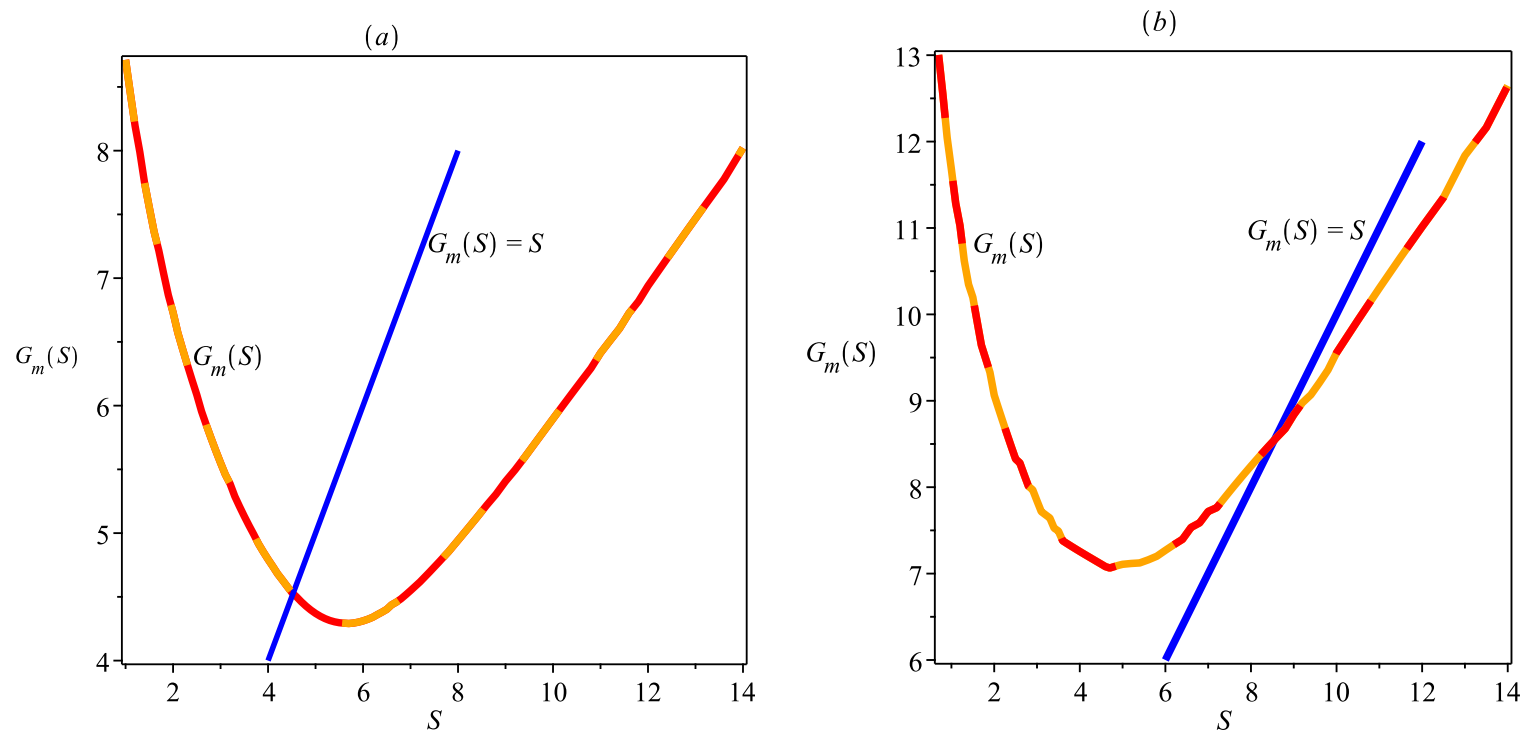

Figure 2: The Poincaré map $G_{m}(S)$ related to the impulsive point series $S$. The parameter values are as follows: $r=1.444, \alpha=0.1, \beta=0.1, d=0.5, a=0.1, b=1, \delta=0.4, \gamma=1$. (c) $a_{1}=0.8$, $b_{1}=0.2, H=16, \theta=0.6, \tau=16$. (b) $H=15, \theta=1, \tau=18$.

For any $u_{k_{1}}^{+}, u_{k_{2}}^{+} \in\left[u_{T}, u^{1}\right)$ and where $u_{k_{1}}^{+}<u_{k_{2}}^{+}$, it is easy to get

$$
\mu\left(u_{k_{1}}^{+}, v_{k_{1}}^{+}\right)<\mu\left(u_{k_{2}}^{+}, v_{k_{1}}^{+}\right)
$$

which implies that there is $G_{m}\left(u_{k_{1}}^{+}\right)<G_{m}\left(u_{k_{2}}^{+}\right)$. Therefore, $G_{m}(S)$ is monotonically increasing on $\left[u_{T}, u^{1}\right)$.

When $u_{k_{1}}^{+}, u_{k_{2}}^{+} \in\left(0, u_{T}\right)$, and satisfy $u_{k_{1}}^{+}<u_{k_{2}}^{+}$. The trajectory $\Gamma_{A_{k_{1}}^{+}}$from point $A_{k_{1}}^{+}\left(u_{k_{1}}^{+}, v_{k_{1}}^{+}\right)$ and the trajectory $\Gamma_{A_{k_{2}}^{+}}$from point $A_{k_{2}}^{+}\left(u_{k_{2}}^{+}, v_{k_{2}}^{+}\right)$will pass through $L_{2}$ and intersect $\Sigma_{N}$ at points $A_{k_{11}}^{+}\left(u_{k_{11}}^{+}, v_{k_{11}}^{+}\right)$and $A_{k_{21}}^{+}\left(u_{k_{21}}^{+}, v_{k_{21}}^{+}\right)$, respectively. Here $u_{k_{i 1}}^{+}(i=1,2) \in\left[u_{T}, u^{1}\right)$ and $u_{k_{11}}^{+}>u_{k_{21}}^{+}$. It can be seen that $G_{m}\left(u_{k_{1}}^{+}\right)>G_{m}\left(u_{k_{2}}^{+}\right)$. Therefore $G_{m}(S)$ is monotonically decreasing on $\left(0, u_{T}\right)$.

(ii) Equation (5) suggests that $w(u, v)$ is continuously differentiable. Thus $G_{m}(S)$ is continuously differentiable in the first quadrant.

(iii) When $G_{m}\left(u_{T}\right)=u_{T}$, then $u_{T}$ is the fixed point of function $G_{m}(S)$.

If $G_{m}\left(u_{T}\right)>u_{T}$, that is $G_{m}\left(u_{T}\right)-u_{T}>0$. Since $0<\delta<1$, there must be a value $S^{*}$ such that

$$
G_{m}\left(S^{*}\right)=S^{*}\left(1-\frac{\delta S^{*}}{S^{*}+\gamma}\right)=S^{*}\left(1-\delta-\frac{\delta \gamma}{S^{*}+\gamma}\right)<S^{*}(1-\delta)<S^{*}
$$

So there is at least one $\tilde{u} \in\left(u_{T}, S^{*}\right)$, satisfying $G_{m}(\tilde{u})=\tilde{u}$.

When $G_{m}\left(u_{T}\right)<u_{T}$, let $G_{m}\left(u_{T}\right)=u_{1}<u_{T}$. We know that $G_{m}(S)$ is monotonically decreasing on $\left(0, u_{T}\right]$, so $G_{m}\left(u_{1}\right)>G_{m}\left(u_{T}\right)=u_{1}$, and because $G_{m}\left(u_{T}\right)<u_{T}$, so there is at least one $\tilde{u} \in\left(u_{1}, u_{T}\right)$ satisfies $G_{m}(\tilde{u})=\tilde{u}$.

From the above analysis, we know that $G_{m}(S)$ has at least one fixed point. Next we prove the uniqueness by contradiction. Assume the system has two fixed points, $\tilde{u}_{1}$ and $\tilde{u}_{2}$ respectively, so 
that $G_{m}\left(\tilde{u}_{1}\right)=\tilde{u}_{1}$ and $G_{m}\left(\tilde{u}_{2}\right)=\tilde{u}_{2}$. Let $\tilde{u}_{1}<\tilde{u}_{2}$, we define

$$
d_{\tilde{u}_{1} \tilde{u}_{2}}(u)=u\left(v, \tilde{u}_{2}\right)-u\left(v, \tilde{u}_{1}\right) .
$$

Differentiate the last equation with respect to $t$. We obtain

$$
\begin{aligned}
d_{\tilde{u}_{1} \tilde{u}_{2}}^{\prime}(u) & =u^{\prime}\left(v, \tilde{u}_{2}\right)-u^{\prime}\left(v, \tilde{u}_{1}\right) \\
= & \frac{r-\alpha v}{v}\left[\frac{\tilde{u}_{2}}{\beta \tilde{u}_{2}-d-\frac{a \tilde{u}_{2}}{b+\tilde{u}_{2}}}-\frac{\tilde{u}_{1}}{\beta \tilde{u}_{1}-d-\frac{a \tilde{u}_{1}}{b+\tilde{u}_{1}}}\right] .
\end{aligned}
$$

Let

$$
g(u)=\frac{u}{\beta u-d-\frac{a u}{b+u}},
$$

then

$$
g^{\prime}(u)=\frac{-d-a\left(\frac{u}{b+u}\right)^{2}}{\left(\beta u-d-\frac{a u}{b+u}\right)^{2}}<0
$$

so

$$
g\left(\tilde{u}_{2}\right)<g\left(\tilde{u}_{1}\right)
$$

that is

$$
d_{\tilde{u}_{1} \tilde{u}_{2}}^{\prime}(u)<0
$$

i.e.

$$
d_{\tilde{u}_{1} \tilde{u}_{2}}\left(a_{1}+b_{1} \beta \sigma\left(u_{k}^{+}\right)-b_{1} d-\frac{a b_{1} \sigma\left(u_{k}^{+}\right)}{b+\sigma\left(u_{k}^{+}\right)}\right)>d_{\tilde{u}_{1} \tilde{u}_{2}}\left(a_{1}+b_{1} \beta u_{k}^{+}-b_{1} d-\frac{a b_{1} u_{k}^{+}}{b+u_{k}^{+}}\right) .
$$

From system (3):

$$
\begin{aligned}
& \tilde{u}_{1}=\mu\left(\tilde{u}_{1}\right)\left(1-\frac{\delta \mu\left(\tilde{u}_{1}\right)}{\mu\left(\tilde{u}_{1}\right)+\gamma}\right) \\
& =\mu\left(\tilde{u}_{1}\right)\left(1-\delta+\frac{\delta \gamma}{\mu\left(\tilde{u}_{1}\right)+\gamma}\right) \\
& =\left[\mu\left(\tilde{u}_{2}\right)-d_{\tilde{u}_{1} \tilde{u}_{2}}\left(a_{1}+b_{1} \beta u_{k}^{+}-b_{1} d-\frac{a b_{1} u_{k}^{+}}{b+u_{k}^{+}}\right)\right]\left(1-\delta+\frac{\delta \gamma}{\mu\left(\tilde{u}_{1}\right)+\gamma}\right) \\
& =\mu\left(\tilde{u}_{2}\right)\left(1-\delta+\frac{\delta \gamma}{\mu\left(\tilde{u}_{1}\right)+\gamma}\right)-d_{\tilde{u}_{1} \tilde{u}_{2}}\left(a_{1}+b_{1} \beta u_{k}^{+}-b_{1} d-\frac{a b_{1} u_{k}^{+}}{b+u_{k}^{+}}\right)\left(1-\delta+\frac{\delta \gamma}{\mu\left(\tilde{u}_{1}\right)+\gamma}\right) \\
& >\mu\left(\tilde{u}_{2}\right)\left(1-\delta+\frac{\delta \gamma}{\mu\left(\tilde{u}_{2}\right)+\gamma}\right)-\frac{\tilde{u}_{1} d_{\tilde{u}_{1}} \tilde{u}_{2}\left(a_{1}+b_{1} \beta u_{k}^{+}-b_{1} d-\frac{a b_{1} u_{k}^{+}}{b+u_{k}^{+}}\right.}{\mu\left(\tilde{u}_{1}\right)} \\
& =\tilde{u}_{2}-\frac{\tilde{u}_{1} d_{\tilde{u}_{1} \tilde{u}_{2}}\left(a_{1}+b_{1} \beta u_{k}^{+}-b_{1} d-\frac{a b_{1} u_{k}^{+}}{b+u_{k}^{+}}\right)}{\mu\left(\tilde{u}_{1}\right)},
\end{aligned}
$$

that is

$$
\frac{\tilde{u}_{1} d_{\tilde{u}_{1} \tilde{u}_{2}}\left(a_{1}+b_{1} \beta u_{k}^{+}-b_{1} d-\frac{a b_{1} u_{k}^{+}}{b+u_{k}^{+}}\right)}{\mu\left(\tilde{u}_{1}\right)}>\tilde{u}_{2}-\tilde{u}_{1}=d_{\tilde{u}_{1} \tilde{u}_{2}}\left(a_{1}+b_{1} \beta \sigma\left(u_{k}^{+}\right)-b_{1} d-\frac{a b_{1} \sigma\left(u_{k}^{+}\right)}{b+\sigma\left(u_{k}^{+}\right)}\right) .
$$


It is easy to know that $\frac{\tilde{u}_{1}}{\mu\left(\tilde{u}_{1}\right)}<1$ if $a_{1}+b_{1} \beta u^{*}-b_{1} d-\frac{a b_{1} u^{*}}{b+u^{*}}<v^{*}$, so

$$
\begin{aligned}
& d_{\tilde{u}_{1} \tilde{u}_{2}}\left(a_{1}+b_{1} \beta u_{k}^{+}-b_{1} d-\frac{a b_{1} u_{k}^{+}}{b+u_{k}^{+}}\right) \\
& >\frac{\tilde{u}_{1} d_{\tilde{u}_{1} \tilde{u}_{2}}\left(a_{1}+b_{1} \beta u_{k}^{+}-b_{1} d-\frac{a b_{1} u_{k}^{+}}{b+u_{k}^{+}}\right)}{\mu\left(\tilde{u}_{1}\right)} \\
& >d_{\tilde{u}_{1} \tilde{u}_{2}}\left(a_{1}+b_{1} \beta \sigma\left(u_{k}^{+}\right)-b_{1} d-\frac{a b_{1} \sigma\left(u_{k}^{+}\right)}{b+\sigma\left(u_{k}^{+}\right)}\right),
\end{aligned}
$$

which is contradictory with

$$
d_{\tilde{u}_{1} \tilde{u}_{2}}\left(a_{1}+b_{1} \beta \sigma\left(u_{k}^{+}\right)-b_{1} d-\frac{a b_{1} \sigma\left(u_{k}^{+}\right)}{b+\sigma\left(u_{k}^{+}\right)}\right)>d_{\tilde{u}_{1} \tilde{u}_{2}}\left(a_{1}+b_{1} \beta u_{k}^{+}-b_{1} d-\frac{a b_{1} u_{k}^{+}}{b+u_{k}^{+}}\right) .
$$

So the fixed point is unique.

When $\frac{H}{a_{1}+b_{1} \beta u^{*}-b_{1} d-\frac{a b_{1} u^{*}}{b+u^{*}}}>v^{*}$ and $\Gamma_{T}$ intersects with $\Sigma_{M}$ (case II), $G_{m}(S)$ of system (3) has similar properties with case I.
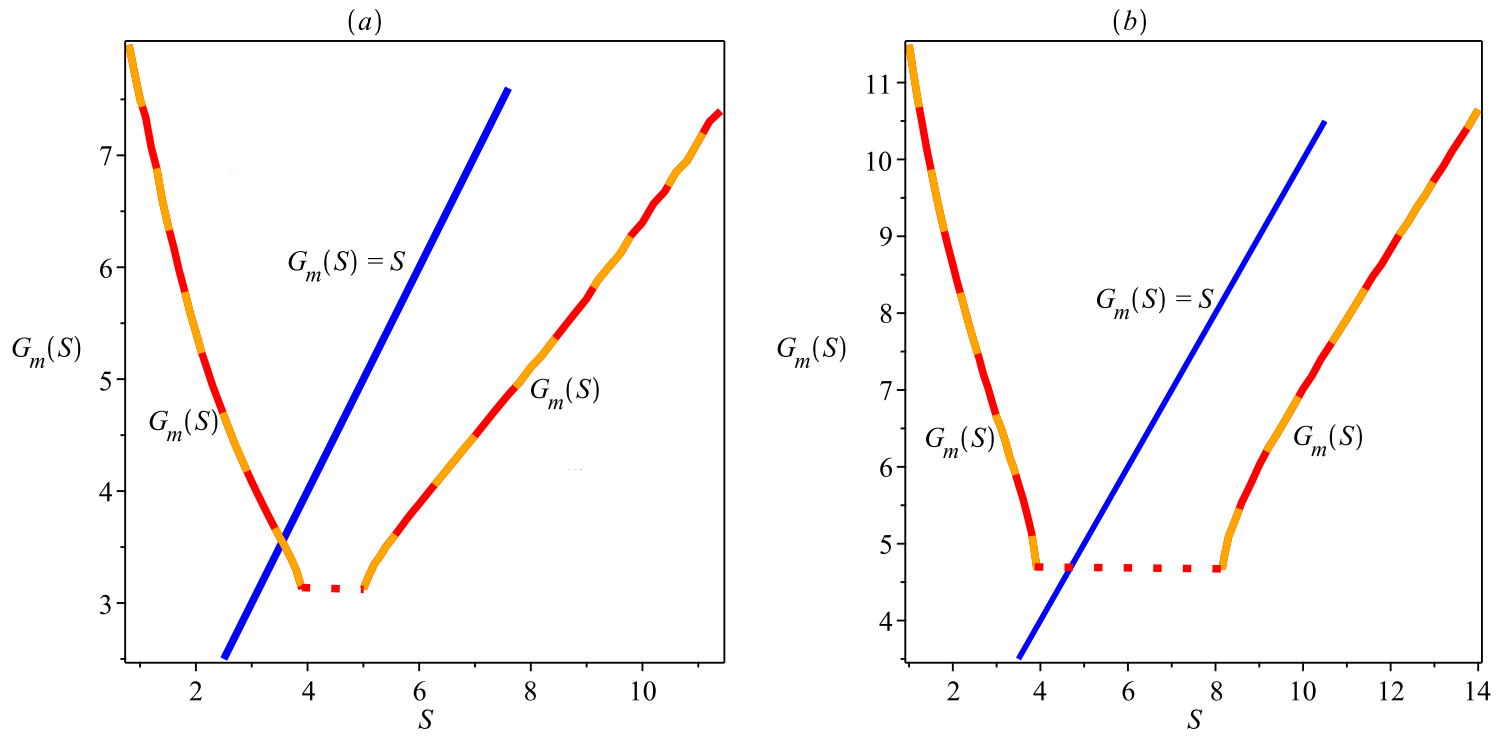

Figure 3: The Poincaré map $G_{m}(S)$ related to the impulsive point series $S$. The parameter values are as follows: $r=1.444, \alpha=0.1, \beta=0.15, d=0.5, a=0.5, b=1, \gamma=1$. (a) $a_{1}=0.6, b_{1}=0.4$, $H=13, \delta=0.7, \theta=1, \tau=18$. (b) $a_{1}=0.8, b_{1}=0.2, H=16, \delta=0.4, \theta=0.6, \tau=65$.

Theorem 3.2. Suppose $\frac{H}{a_{1}+b_{1} \beta u^{*}-b_{1} d-\frac{a b_{1} u^{*}}{b+u^{*}}}>v^{*}$. Then, then trajectory $\Gamma_{W^{-}}$is tangent to $\Sigma_{M}$ at $W$ and intersect $\Sigma_{N}$ at point $P_{1}\left(u_{P_{1}}, v_{P_{1}}\right)$ and point $P_{2}\left(u_{P_{2}}, v_{P_{2}}\right)$, respectively, where $u_{P_{1}}>u_{P_{2}}$. Furthermore, $G_{m}(S)$ has the following properties:

(i) The domain of $G_{m}(S)$ is $\left(0, u_{P_{2}}\right] \cup\left[u_{P_{1}}, u^{1}\right)$, and $G_{m}(S)$ is monotonically decreasing on $\left(0, u_{P_{2}}\right]$ and monotonically increasing on $\left[u_{P_{1}}, u^{1}\right)$.

(ii) When $G_{m}\left(u_{P_{2}}\right) \leq u_{P_{2}}$, there is a unique fixed point on $\left(0, u_{P_{2}}\right]($ see Fig. 3(a)). When $G_{m}\left(u_{P_{2}}\right)>u_{P_{2}}$, there is no fixed point(see Fig. 3(b)). 
Proof. (i) Because $E^{*}\left(u^{*}, v^{*}\right)$ is the center point, if $\frac{H}{a_{1}+b_{1} \beta u^{*}-b_{1} d-\frac{a b_{1} u^{*}}{b+u^{*}}}>v^{*}$ and the trajectory $\Gamma_{W^{-}}$is tangent to $\Sigma_{M}$ at $W$ and intersect the $\Sigma_{N}$ at point $P_{1}\left(u_{P_{1}}, v_{P_{1}}\right)$ and point $P_{2}\left(u_{P_{2}}, v_{P_{2}}\right)$, respectively, where $u_{P_{1}}>u_{P_{2}}$. Then take any point $A_{k}^{+}\left(u_{k}^{+}, v_{k}^{+}\right)$in $\Sigma_{N}$, if $u_{k}^{+} \in\left(0, u_{P_{2}}\right] \cup\left[u_{P_{1}},+\infty\right)$, the trajectory $\Gamma_{A_{k}^{+}}$will reach the $M_{3}$ at point $A_{k+1}\left(u_{k}, v_{k}\right)$, if $u_{k}^{+} \in\left(u_{P_{2}}, u_{P_{1}}\right)$ the $\Gamma_{A_{k}^{+}}$has no intersection with the $M_{3}$. So the domain of $G_{m}(S)$ is $\left(0, u_{P_{2}}\right] \cup\left[u_{P_{1}}, u^{1}\right)$.

For any $u_{k_{1}}^{+}, u_{k_{2}}^{+} \in\left[u_{P_{1}}, u^{1}\right)$ and $u_{k_{1}}^{+}<u_{k_{2}}^{+}$, easy to get

$$
\mu\left(u_{k_{1}}^{+}, v_{k_{1}}^{+}\right)<\mu\left(u_{k_{2}}^{+}, v_{k_{2}}^{+}\right)
$$

so $G_{m}\left(u_{k_{1}}^{+}\right)<G_{m}\left(u_{k_{2}}^{+}\right)$. Therefore $G_{m}(S)$ is monotonically increasing on $\left[u_{P_{1}}, u^{1}\right)$.

When $u_{k_{1}}^{+}, u_{k_{2}}^{+} \in\left(0, u_{P_{2}}\right]$, where $u_{k_{1}}^{+}<u_{k_{2}}^{+}$. The trajectory from $A_{k_{1}}^{+}\left(u_{k_{1}}^{+}, v_{k_{1}}^{+}\right)$and $A_{k_{2}}^{+}\left(u_{k_{2}}^{+}, v_{k_{2}}^{+}\right)$ will pass through the $L_{2}$ intersect the $\Sigma_{N}$ at point $A_{k_{11}}^{+}\left(u_{k_{11}}^{+}, v_{k_{11}}^{+}\right)$and point $A_{k_{21}}^{+}\left(u_{k_{21}}^{+}, v_{k_{21}}^{+}\right)$, respectively. Then $u_{k_{i 1}}^{+}(i=1,2) \in\left[u_{32}, u^{1}\right)$ and $u_{k_{11}}^{+}>u_{k_{21}}^{+}$. So $G_{m}\left(u_{k_{1}}^{+}\right)>G_{m}\left(u_{k_{2}}^{+}\right)$, therefore $G_{m}(S)$ is monotonically decreasing on $\left(0, u_{P_{2}}\right]$.

(ii) Consider the relationship between $G_{m}\left(u_{P_{2}}\right)$ and $u_{P_{2}}$ :

(a) When $G_{m}\left(u_{P_{2}}\right) \leq u_{P_{2}}$ (see Fig. 3(a)), we assume $G_{m}\left(u_{P_{2}}\right)=u_{1} \leq u_{P_{2}}$, we know that $G_{m}(S)$ is monotonically decreasing on $\left(0, u_{P_{2}}\right]$, so $G_{m}\left(u_{P_{2}}\right) \geq G_{m}\left(u_{P_{2}}\right)=u_{1}$, and because $G_{m}\left(u_{P_{2}}\right) \leq u_{P_{2}}$, so there is a point $\tilde{u} \in\left(u_{1}, u_{P_{2}}\right]$ and satisfies $G_{m}(\tilde{u})=\tilde{u}$.

(b) When $G_{m}\left(u_{P_{2}}\right)>u_{P_{2}}$ (see Fig. $\left.3(\mathrm{~b})\right)$, there is no $\tilde{u} \in\left(0, u_{P_{2}}\right]$ satisfies $G_{m}(\tilde{u})=\tilde{u}$.

For any $u_{k} \in\left(u_{P_{1}}, u^{1}\right)$, The trajectory of point $A_{k}\left(u_{k}, v_{k}\right)$ on $\Sigma_{N}$ is tangent to the $\Sigma_{M}$ at point $A_{k}^{+}\left(u_{k}^{+}, v_{k}^{+}\right), A_{k}^{+}\left(u_{k}^{+}, v_{k}^{+}\right)$will be pulsed to $A_{k+1}\left(u_{k+1}, v_{k+1}\right)$. Easy to get $u_{k+1}<u_{k}^{+}<u_{k}$, that is $u_{k+1} \neq u_{k}$, so there is no $\tilde{u} \in\left(u_{P_{1}}, u^{1}\right)$ and satisfies $G_{m}(\tilde{u})=\tilde{u}$.

\section{The order- $k(k \geq 1)$ periodic solution of the semi-continuous dynamic system (3) and its stability}

Theorem 3.1 has proved that system (3) has a fixed point under certain conditions, that is, the system has an order-1 periodic solution. Below we will give more properties of the order-1 solution, and also the existence of the order- $k$ solutions.

Theorem 4.1. The order-1 periodic solution of system (3) is globally asymptotically stable if $\frac{H}{a_{1}+b_{1} \beta u^{*}-b_{1} d-\frac{a b_{1} u^{*}}{b+u^{*}}}<v^{*}$ and $G_{m}\left(u_{Q_{1}}\right)>u_{Q_{1}}$.

Proof. When $G_{m}\left(u_{Q_{1}}\right)>u_{Q_{1}}, G_{m}(S)$ has a fixed point $\tilde{u}$ on $\left(u_{Q_{1}}, u^{1}\right)$, that is, $G_{m}(\tilde{u})=\tilde{u}$ from the (iii) of Theorem 3.1. For any point $A_{0}^{+}\left(u_{0}^{+}, v_{0}^{+}\right)$in $\Sigma_{N}$, where $u_{0}^{+}>u_{Q_{1}}, \Gamma_{A_{0}^{+}}$will intersect $\Sigma_{M}$ at the point $p_{1}^{+}\left(u_{1}^{+}, v_{1}^{+}\right)$, which is $G_{m}\left(u_{0}^{+}\right)=u_{1}^{+}$, repeating the above process,

$$
G_{m}\left(G_{m}\left(u_{0}^{+}\right)\right)=G_{m}^{2}\left(u_{0}^{+}\right),
$$

that is,

$$
G_{m}\left(u_{1}^{+}\right)=u_{2}^{+},
$$


further available,

$$
u_{n}^{+}=G_{m}^{n}\left(u_{0}^{+}\right), n=1,2, \cdots
$$

Then we have the following three cases:

Case 1: $u_{Q_{1}}<u_{0}^{+} \leq \tilde{u}$. Since $G_{m}\left(u_{Q_{1}}\right)>u_{Q_{1}}$ and $G_{m}(S)$ is monotonously increasing on $\left(u_{Q_{1}},+\infty\right)$, letting $G_{m}\left(u_{i}^{+}\right)=u_{i+1}^{+}$yields

$$
\begin{aligned}
& u_{0}^{+}<G_{m}\left(u_{0}^{+}\right)=u_{1}^{+} \leq G_{m}(\tilde{u})=\tilde{u}, \\
& u_{0}^{+}<G_{m}\left(u_{0}^{+}\right)<G_{m}\left(u_{1}^{+}\right)=G_{m}{ }^{2}\left(u_{0}^{+}\right) \leq G_{m}(\tilde{u})=\tilde{u},
\end{aligned}
$$

Following the same fashion, we obtain that

$$
u_{0}^{+}<G_{m}\left(u_{0}^{+}\right)<\ldots<G_{m}^{n}\left(u_{0}^{+}\right)<\ldots<\tilde{u} .
$$

Then we can get

$$
\lim _{n \rightarrow+\infty} G_{m}^{n}\left(u_{0}^{+}\right)=\tilde{u} .
$$

Case 2: $\tilde{u}<u_{0}^{+}<u^{1}$. In this case, we have

$$
\tilde{u}=G_{m}(\tilde{u})<G_{m}\left(u_{0}^{+}\right),
$$

and

$$
\tilde{u}=G_{m}(\tilde{u})<G_{m}^{2}\left(u_{0}^{+}\right)<G\left(u_{0}^{+}\right) .
$$

By mathematical induction,

$$
\tilde{u}=G_{m}(\tilde{u})<\ldots<G_{m}^{n}\left(u_{0}^{+}\right)<G_{m}^{n-1}\left(u_{0}^{+}\right)<\cdots
$$

Thus,

$$
\lim _{n \rightarrow+\infty} G_{m}^{n}\left(u_{0}^{+}\right)=\tilde{u} .
$$

Case 3: $0<u_{0}^{+}<u_{Q_{1}}$. Since $G_{m}\left(u_{Q_{1}}\right)>u_{Q_{1}}$ and $G_{m}(S)$ is decreasing on $\left(0, u_{Q_{1}}\right)$, we obtain that $G_{m}\left(u_{0}^{+}\right)>G_{m}\left(u_{Q_{1}}\right)>u_{Q_{1}}$ for any $u_{0}^{+} \in\left(0, u_{Q_{1}}\right)$. So we can conclude that $G_{m}\left(u_{0}^{+}\right)>u_{Q_{1}}$. When $u_{Q_{1}}<G_{m}\left(u_{0}^{+}\right)<\tilde{u}$, this is the situation in Case 1 ; when $G_{m}\left(u_{0}^{+}\right)>\tilde{u}$, this is Case 2 above.

Thus we always have

$$
\lim _{n \rightarrow+\infty} G_{m}^{n}\left(u_{0}^{+}\right)=\tilde{u}
$$

The conclusion is proved.

Theorem 4.2. The semi-continuous dynamical system (3) has a stable order-1 periodic solution or an order-2 periodic solution when $\frac{H}{a_{1}+b_{1} \beta u^{*}-b_{1} d-\frac{a b_{1} u^{*}}{b+u^{*}}}<v^{*}, G_{m}\left(u_{Q_{1}}\right)<u_{Q_{1}}$ and $G_{m}^{2}\left(u_{Q_{1}}\right)<u_{Q_{1}}$. 
Proof. Take a point $A_{0}^{+}\left(u_{0}^{+}, v_{0}^{+}\right)$on $\Sigma_{N}$. Since $E^{*}$ is a center point, $\Gamma_{A_{0}^{+}}$will intersect $\Sigma_{M}$ at point $A_{1}\left(u_{1}, v_{1}\right)$, where $u_{1}=\mu\left(u_{0}^{+}, v_{0}^{+}\right)$. $p_{1}$ will reach point $p_{1}^{+}\left(u_{1}^{+}, v_{1}^{+}\right)$by an impulse, so $G_{m}\left(u_{0}^{+}\right)=u_{1}^{+}$. Repeat the above process

$$
u_{n}^{+}=G_{m}^{n}\left(u_{0}^{+}\right)=G_{m}\left(G_{m}^{n-1}\left(u_{0}^{+}\right)\right), \quad(n=1,2, \cdots),
$$

when $u_{Q_{1}} \leq u_{0}^{+}<u^{1}, G_{m}(S)$ is increasing on $\left[u_{Q_{1}}, u^{1}\right)$ and from the (i) of Theorem 3.1 we know there is no fixed point on $\left[u_{Q_{1}}, u^{1}\right)$. Therefore, there is a positive integer $i$ that satisfies

$$
u_{i-1}^{+}=G_{m}\left(u_{i-2}^{+}\right)<u_{Q_{1}}
$$

and

$$
u_{i}^{+}=G_{m}\left(u_{i-1}^{+}\right)<G_{m}\left(u_{Q_{1}}\right)<u_{Q_{1}} .
$$

When $0<u_{0}^{+}<u_{Q_{1}}$, the $\Gamma_{A_{0}^{+}}$will pass through the $L_{2}$ and intersects the $\Sigma_{N}$ at $p_{0}^{\prime}\left(u_{0}^{\prime}, H-\frac{\tau}{1+\theta H}\right)$, where $0<u_{0}^{\prime}<u_{Q_{1}}$, this can be transformed into the above situation.

So for any $u_{0}^{+} \in\left(0, u^{1}\right)$, there is always be a $i$ satisfies

$$
G_{m}\left(u_{Q_{1}}\right)<G_{m}^{i}\left(u_{0}^{+}\right)<u_{Q_{1}}(i \geq 1) .
$$

So we just consider the initial point $p_{0}^{+}\left(u_{0}^{+}, v_{0}^{+}\right)$, where $u_{Q_{1}} \leq u_{0}^{+}<G_{m}\left(u_{Q_{1}}\right)$. Since $G_{m}(S)$ is monotonically decreasing on $\left[G_{m}\left(u_{Q_{1}}\right), u_{Q_{1}}\right]$, we have

$$
G_{m}\left[G_{m}\left(u_{Q_{1}}\right), u_{Q_{1}}\right] \subset\left[G_{m}\left(u_{Q_{1}}\right), u_{Q_{1}}\right]
$$

Let $G_{m}\left(u_{0}^{+}\right) \neq u_{0}^{+}$and $G_{m}^{2}\left(u_{0}^{+}\right) \neq u_{0}^{+}$. Consider the following four situations:

Case I: $u_{Q_{1}} \geq u_{0}^{+}>G_{m}^{2}\left(u_{0}^{+}\right)>G_{m}\left(u_{0}^{+}\right) \geq G_{m}\left(u_{Q_{1}}\right)$. According to the monotonicity of $G_{m}(S)$ :

$$
u_{2}^{+}=G_{m}\left(u_{1}^{+}\right)>G_{m}\left(u_{2}^{+}\right)=u_{3}^{+}>G_{m}\left(u_{0}^{+}\right)=u_{1}^{+},
$$

furthermore,

$$
u_{4}^{+}=G_{m}\left(u_{3}^{+}\right)<G_{m}\left(u_{0}^{+}\right)=u_{0}^{+} .
$$

Thus there is

$$
u_{Q_{1}} \geq u_{0}^{+}>u_{2}^{+}>u_{4}^{+}>u_{3}^{+}>u_{1}^{+} \geq G_{m}\left(u_{Q_{1}}\right) .
$$

Proved by mathematical induction:

$$
u_{Q_{1}} \geq u_{0}^{+}>u_{2}^{+}>\ldots>u_{2 n}^{+}>u_{2 n+2}^{+}>\ldots>u_{2 n+1}^{+}>u_{2 n-1}^{+}>\ldots>u_{1}^{+} \geq G_{m}\left(u_{Q_{1}}\right) .
$$

Case II: $u_{Q_{1}} \geq u_{1}^{+}>u_{0}^{+}>u_{2}^{+} \geq G_{m}\left(u_{Q_{1}}\right) . G_{m}(S)$ is monotonically decreasing on $\left[G_{m}\left(u_{Q_{1}}\right), u_{Q_{1}}\right]$. So

$$
u_{3}^{+}=G_{m}\left(u_{2}^{+}\right)>G_{m}\left(u_{0}^{+}\right)=u_{1}^{+}>G_{m}\left(u_{1}^{+}\right)=u_{2}^{+},
$$


and

$$
u_{4}^{+}=G_{m}\left(u_{3}^{+}\right)<G_{m}\left(u_{1}^{+}\right)=u_{2}^{+}<G_{m}\left(u_{2}^{+}\right)=u_{3}^{+},
$$

then

$$
u_{Q_{1}} \geq u_{3}^{+}>u_{1}^{+}>u_{0}^{+}>u_{2}^{+}>u_{4}^{+} \geq G\left(u_{Q_{1}}\right),
$$

SO

$u_{Q_{1}} \geq \ldots>u_{2 n+1}^{+}>u_{2 n-1}^{+}>\ldots>u_{3}^{+}>u_{1}^{+}>u_{0}^{+}>u_{2}^{+}>u_{4}^{+}>\ldots>u_{2 n}^{+}>\ldots \geq G\left(u_{Q_{1}}\right)$.

Case III: $u_{Q_{1}} \geq u_{1}^{+}>u_{2}^{+}>u_{0}^{+} \geq G_{m}\left(u_{Q_{1}}\right)$.

Easy to get

$$
u_{Q_{1}} \geq u_{1}^{+}>u_{3}^{+}>\ldots>u_{2 n-1}^{+}>\ldots>u_{2 n+1}^{+}>u_{2 n}^{+}>\ldots>u_{2}^{+}>u_{0}^{+} \geq G_{m}\left(u_{Q_{1}}\right) .
$$

Case IV: $u_{Q_{1}} \geq u_{2}^{+}>u_{0}^{+}>u_{1}^{+} \geq G_{m}\left(u_{Q_{1}}\right)$.

This situation is similar to Case I, it can get

$$
G_{m}\left(u_{Q_{1}}\right) \leq u_{2 n+1}^{+}<u_{2 n-1}^{+}<\ldots<u_{1}^{+}<u_{0}^{+}<u_{2}^{+}<\ldots<u_{2 n}^{+}<u_{2 n+1}^{+}<\ldots \leq u_{Q_{1}} .
$$

For Case I and Case III, there is $\tilde{u} \in\left(u_{Q_{1}}, G_{m}\left(u_{Q_{1}}\right)\right)$, so that

$$
\lim _{n \rightarrow \infty} u_{2 n}^{+}=\lim _{n \rightarrow \infty} u_{2 n-1}^{+}=\tilde{u}
$$

That is to say, in these two cases, system (3) has a stable order-1 periodic solution.

For case II and case IV, $\tilde{u_{1}} \neq \tilde{u_{2}}$ and

$$
\begin{array}{r}
\lim _{n \rightarrow \infty} u_{2 n-1}{ }^{+}=\tilde{u}_{1}, \\
\lim _{n \rightarrow \infty} u_{2 n}{ }^{+}=\tilde{u}_{2},
\end{array}
$$

That is to say system (3) has a stable order-2 periodic solution in these two cases.

Theorem 4.3. Let $H_{1}<u_{Q_{1}}$ and $G_{m}\left(u_{Q_{1}}\right)<u_{Q_{1}}$. Then the necessary and sufficient condition for the order-1 periodic solution of system (3) to be globally stable is $G_{m}^{2}\left(u^{+}\right)<u^{+}$for any $u^{+} \in$ $\left(0, u_{Q_{1}}\right]$.

Proof. Sufficiency:

When $G_{m}\left(u_{Q_{1}}\right)<u_{Q_{1}}$, there exists $\tilde{u} \in\left(G_{m}\left(u_{Q_{1}}\right), u_{Q_{1}}\right)$ which satisfies $G_{m}(\tilde{u})=\tilde{u}$.

For any $u^{+} \in\left(\tilde{u}, u_{Q_{1}}\right)$, we make $u_{1}^{+}=G_{m}\left(u^{+}\right)$and $u_{2}^{+}=G_{m}\left(u_{1}^{+}\right)=G_{m}^{2}\left(u^{+}\right)$, since $G_{m}^{2}\left(u^{+}\right)<$ $u^{+}<u_{Q_{1}}$, easy to get $\tilde{u}>u_{1}^{+}>G_{m}\left(u_{Q_{1}}\right)$, further more, $u_{Q_{1}}>u_{2}^{+}>u_{4}^{+}>\tilde{u}>u_{3}^{+}>u_{1}^{+}>$ $G_{m}\left(u_{Q_{1}}\right)$, so $u_{Q_{1}}>u_{2 n}^{+}>\tilde{u}>u_{2 n+1}^{+}>G_{m}\left(u_{Q_{1}}\right)$.

So

$$
\lim _{n \rightarrow \infty} u_{2 n}^{+}=\lim _{n \rightarrow \infty} u_{2 n+1}^{+}=\tilde{u}
$$


Necessity: If $G_{m}^{2}\left(u^{+}\right)<u^{+}$is not true for any $u^{+} \in\left[\tilde{u}, u_{Q_{1}}\right]$, there exists a maximum $u_{0} \in$ $\left[\tilde{u}, u_{Q_{1}}\right]$ and satisfies $G_{m}{ }^{2}\left(u_{0}\right) \geq u_{0}$. There exist $u_{1}$ and $G_{m}^{2}\left(u_{1}\right)<u_{1}$ for any $\tilde{u}-\varepsilon<u_{1}<\tilde{u}+\varepsilon$, where $\varepsilon>0$. From the continuity of $G_{m}^{2}(u)$ on the $\left[u_{0}, u_{1}\right]$ that there is at least one $\vec{u} \in\left[u_{0}, u_{1}\right]$ and $G_{m}{ }^{2}(\vec{u})=\vec{u}$. this is contradictory.

Theorem 4.4. If $G_{m}\left(u_{Q_{1}}\right)<u_{Q_{1}}$ and there is $u_{m}^{+}$such that $u_{m}^{+}=\min \left\{u^{+}: G_{m}\left(u^{+}\right)=u_{Q_{1}}\right\}$, the system (3) has a order-3 periodic solution if $G_{m}^{2}\left(u_{Q_{1}}\right)<u_{m}^{+}$.

Proof. If $G_{m}\left(u_{Q_{1}}\right)<u_{Q_{1}}$, there is a order-1 periodic solution in $\left(G_{m}\left(u_{Q_{1}}\right), u_{Q_{1}}\right)$, i.e. $G_{m}(\tilde{u})=\tilde{u}$, here $\tilde{u} \in\left(G_{m}\left(u_{Q_{1}}\right), u_{Q_{1}}\right)$. Since $G_{m}(\tilde{u})=\tilde{u}$, so there is $u_{m}^{+}$such that $u_{m}^{+} \in(0, \tilde{u})$ and $G_{m}\left(u_{m}^{+}\right)=u_{Q_{1}}$. Further, $G_{m}^{3}\left(u_{m}^{+}\right)=G_{m}^{2}\left(u_{Q_{1}}\right)<u_{m}^{+}$, on the other hand, $\lim _{x \rightarrow 0} G_{m}^{3}(x)>x$, so there is at least one value of $\vec{u}$ such that $G_{m}{ }^{3}(\vec{u})=\vec{u}$.

Remark 4.1. Using the similar arguments, we can show that if $G_{m}^{k-1}\left(u_{Q_{1}}\right)<u_{m}^{+}$, where $G_{m}\left(u_{m}^{+}\right)=$ $u_{Q_{1}}$, then the system (3) has a order- $k(k \geq 2)$ periodic solution.

\section{Simulations and discussions.}

In this section, we carry out some numerical simulations, illustrating our theoretical findings in previous sections, and then discuss these results from biological point of view.

Figure 4(a) is the trajectory of the order-2 periodic solution of the model; Figure 4(b) and Figure 4(c) are the time series of phytoplankton density and fish density under the simulated order-2 periodic solution, respectively. The existence of the order- 2 periodic solution of the system suggests that the rational fishing activities can make the system exist a benign ecological balance. 

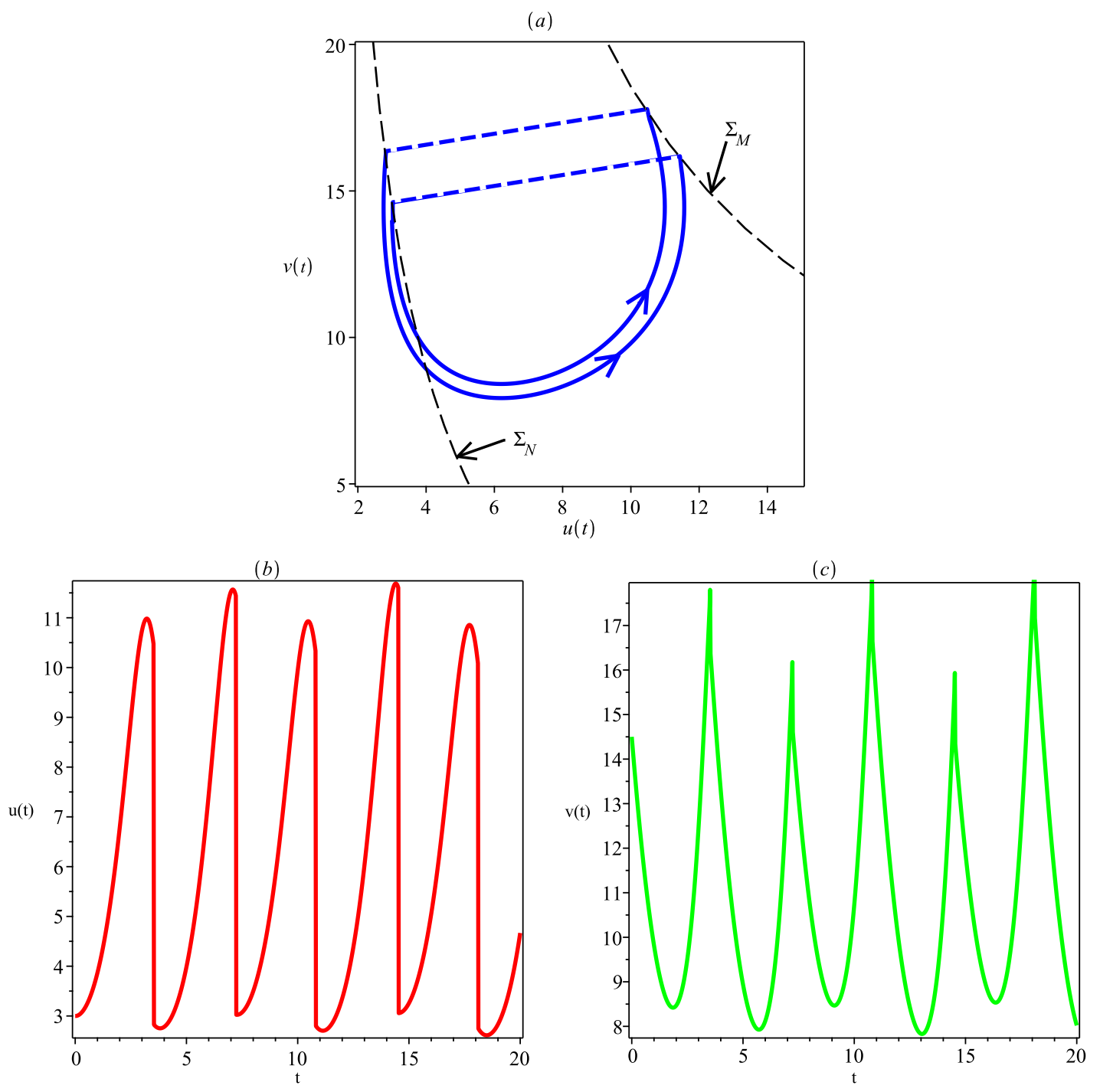

Figure 4: The order-2 periodic solution of Cases III. 
(a)

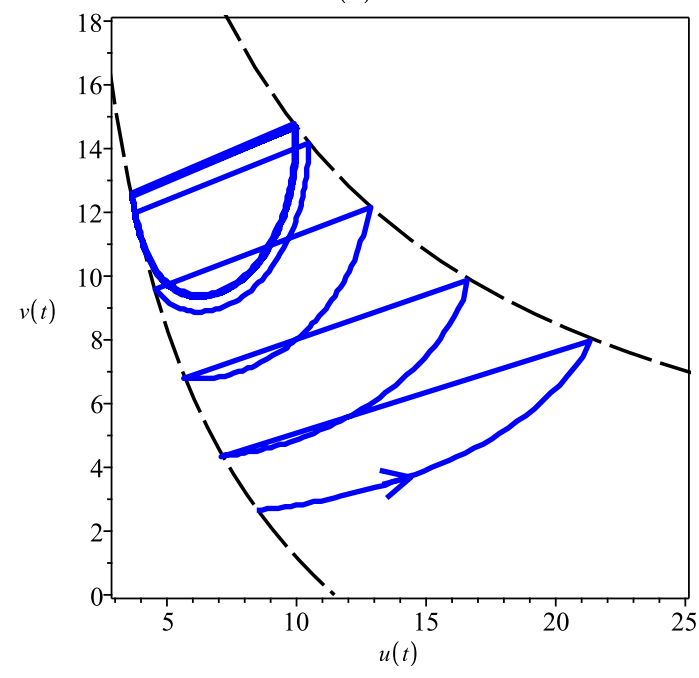

(b)

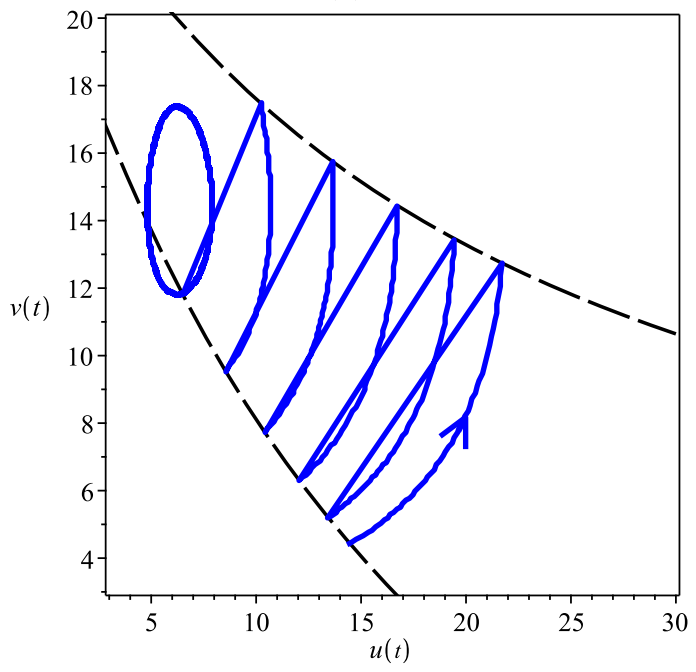

Figure 5: Periodic solutions in the case of Figures 3 a and b, respectively.

In figure 5, we numerically illustrate our mathematical findings in Theorem 3.2, corresponding to the cases in Fig. 3. In sub-figure a, we see the only order-1 periodic solution (see Fig. 5a), and in the case of Figure 3b, there is no order-1 periodic solution (see Fig. 5b).

Fig. 6 illustrates the impulse and phase sets can change significantly with the weighting parameters $a_{1}$ and $b_{1}$. That is to say, the comprehensive fishing measures that comprehensively consider the fish population density and the current fish growth rate are compared to the fishing measures that only consider the fish population density. The model will generate more complex pulse sets and phase sets. Below we discuss which of the two fishing measures is more biologically appropriate. 
(a) $a_{1}=1, b_{1}=0$

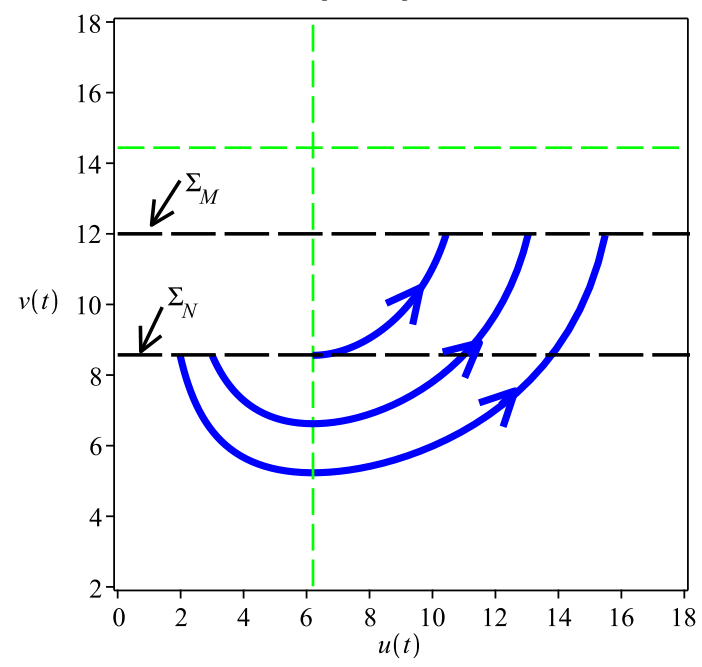

(c) $a_{1}=0.6, b_{1}=0.4$

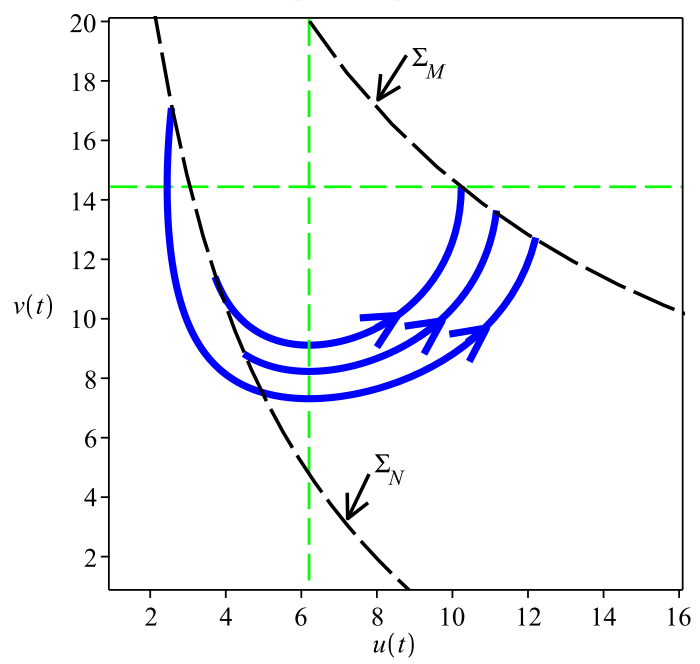

(b) $a_{1}=0.8, b_{1}=0.2$
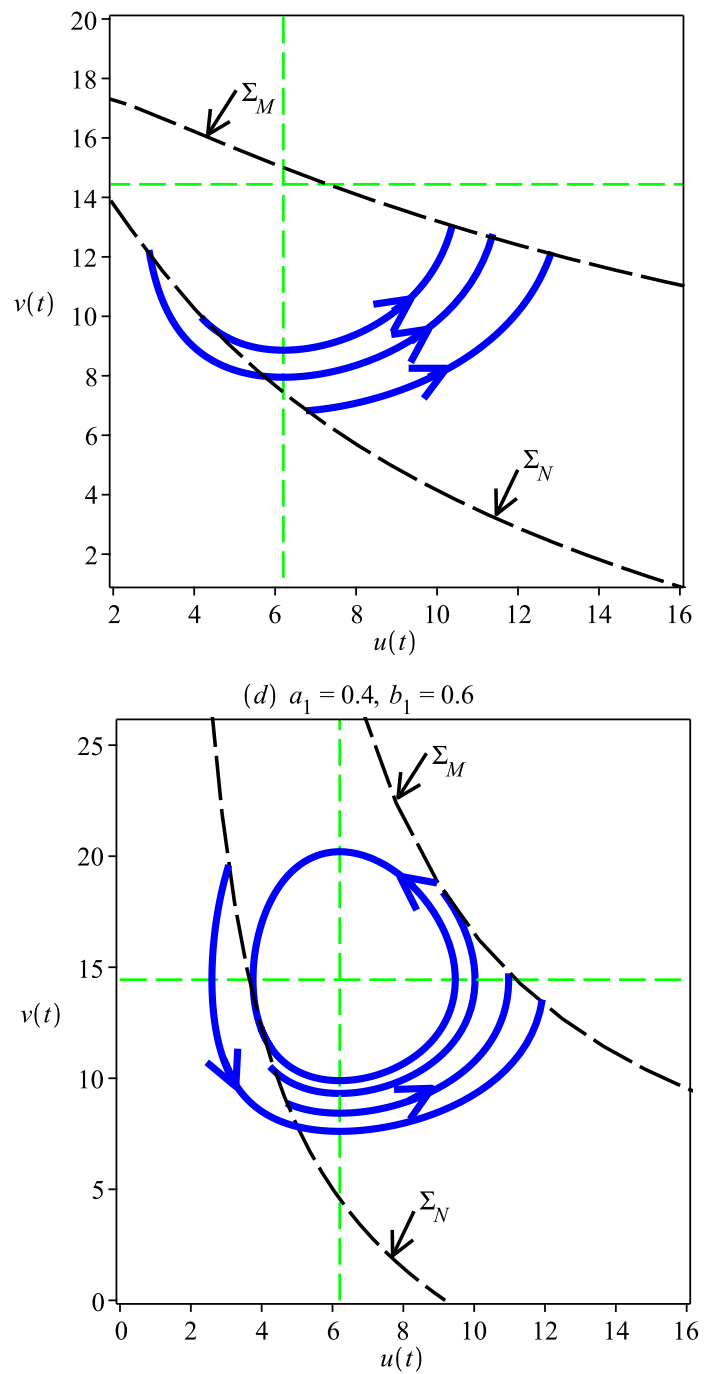

Figure 6: The impulse and phase sets of the system (3) with different weighted parameters $a_{1}$ and $b_{1}$. 


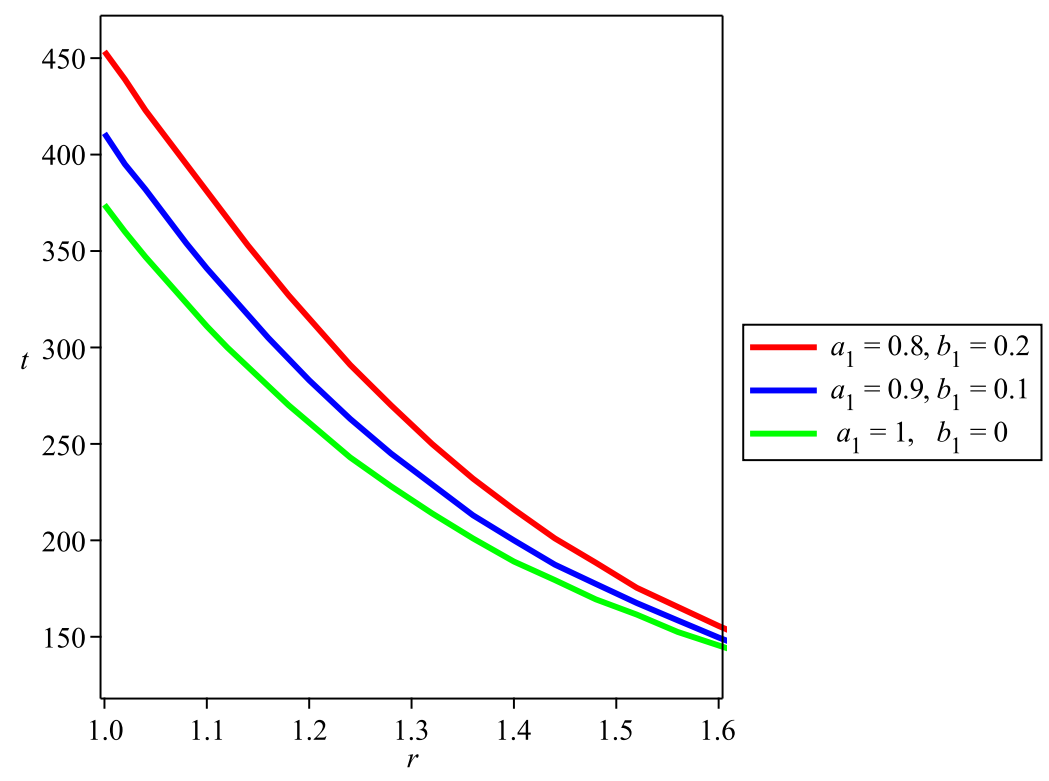

Figure 7: The time $t$ of a single period of the order-1 periodic solution with the growth rate $r$ of phytoplankton.

From Fig. 7) we see that no matter what the value of $r$ is, the smaller the weight of $a_{1}$, the greater the weight of $b_{1}$, the longer the single period of the order-1 periodic solution, that is, the comprehensive control measures that take into account the fish population density and the current growth rate are longer than the cycle time of the system that only considers the fish population density. That is, the comprehensive fishing measures that comprehensively consider the fish population density and the current fish growth rate are more reasonable than the fishing measures that only consider the fish population density.

It can be seen from Figure 8 that the trajectories of different initial points eventually are stabilised. This illustrates the global asymptotic stability of the order-1 periodic solution. This provides theoretical support for the application of state-dependent feedback control in ecosystem balance. 


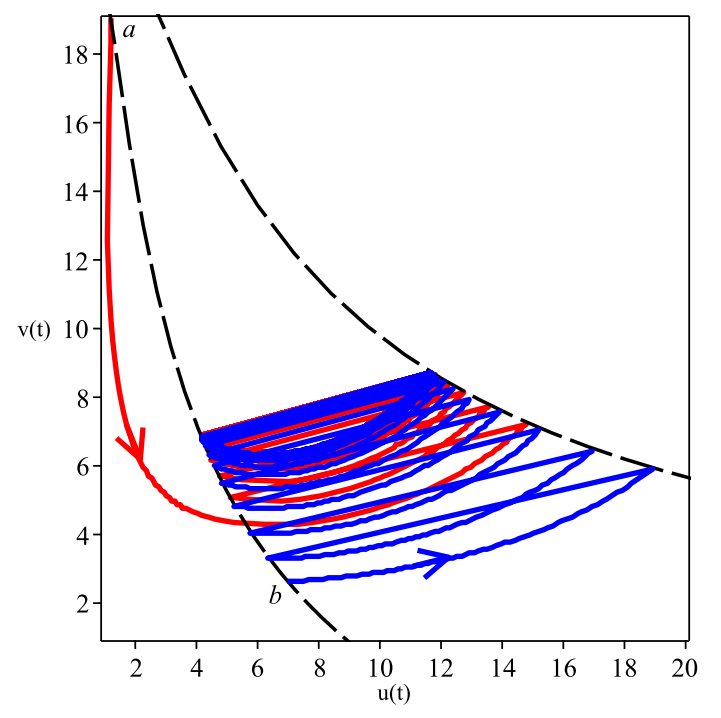

Figure 8: The path curve of system 3 starting from the points $a$ and $b$.

\section{Conclusion}

We establish a phytoplankton-fish model with the impulsive feedback control depending on the density and rate of change of the fish. We have further studied the dynamics of the model, and numerical simulations show that the comprehensive fishing measures that consider the density of fish populations and the current growth rate of fish are more reasonable than the fishing measures that only consider the density of fish populations.

The results of this paper are an extension of literature [31], and we believe that the innovation of this model is to adopt dynamic thresholds determined by fish population density and current fish growth rate. This makes the pulse set and the phase set into two complex curves, which makes it difficult to study the dynamic properties of the model. With the help of Poincaré map, this paper gives a method to prove the existence and stability of periodic solutions of order-1 and the existence of periodic solutions of order- $k(k \geq 2)$. In a biological sense, the order- $k(k \geq 1)$ periodic solution of the state impulse feedback control system shows that under certain conditions, the phytoplankton population density and fish population density can maintain periodic oscillations, in other words, the population size can be controlled within a stable range. Numerical simulation of $a_{1}, b_{1}$, the influence of parameter changes on cycle changes. When $a_{1}=1, b_{1}=0$, the action threshold only depends on the fish density. This is the situation studied in the literature [31], which is a special case of this article.

The analysis method proposed in this paper also plays an important role in the analysis of pulse models with complex phase sets or impulse sets. 


\section{Acknowledgements}

The paper was supported by the National Natural Science Foundation of China (No. 11872335).

\section{Conflict of Interest:}

The authors declare that they have no conflict of interest.

\section{Data availability statement}

The author declares that the data supporting the results of this study are available in the article.

\section{References}

[1] H. Sun, Y. Tian, Continuously harvesting of a phytoplankton-zooplankton system with Holling I functional response, Applied Mechanics Materials 595 (2014) 277-282.

[2] Y. Wang, W. Jiang, H. Wang, Stability and global hopf bifurcation in toxic phytoplankton zooplankton model with delay and selective harvesting, Nonlinear Dynamics 73 (1) (2013) 881-896.

[3] M. P. Sissenwine, J. G. Shepherd, An alternative perspective on recruitment overfishing and biological reference points, Can.j.fish.aquat.sci 44 (4) (1987) 913-918.

[4] S. A. Khamis, J. M. Tchuenche, M. Lukka, M. Heilioe, Dynamics of fisheries with prey reserve and harvesting, International Journal of Computer Mathematics 88 (7-9) (2011) 1776-1802.

[5] M. R. Garvie, C. Trenchea, Optimal control of a nutrient-phytoplankton-zooplankton-fish system, Siam Journal on Control and Optimization 46 (3) (2007) 775-791.

[6] W. Zheng, J. Sugie, Global asymptotic stability and equiasymptotic stability for a timevarying phytoplankton-zooplankton-fish system, Nonlinear Analysis: Real World Applications 46 (3) (2019) 116-136.

[7] U. Srinivasan, W. Cheung, R. Watson, Food security implications of global marine catch losses due to overfishing, Journal of Bioeconomics 12 (3) (2010) 183-200.

[8] R. G. Marcus, T. Catalin, Optimal control of a nutrient-phytoplankton-zooplankton-fish system, Siam Journal on Control Optimization 46 (3) (2007) 775-791.

[9] X. Y. Meng, Y. Q. Wu, Bifurcation and control in a singular phytoplankton-zooplankton-fish model with nonlinear fish harvesting and taxation, International Journal of Bifurcation Chaos 28 (3) (2018) 1850042. 
[10] T. Zhang, X. Liu, X. Meng, T. Zhang, Spatio-temporal dynamics near the steady state of a planktonic system, Computers and Mathematics with Applications 75 (12) (2018) 4490-4504.

[11] S. Sun, C. Guo, Dynamics of a beddington-deangelis type predator-prey model with impulsive effect, Journal of Mathematics 2013 (1) (2013) 1-11.

[12] X. Liu, L. Chen, Extinction and permanence of a general predator-prey system with impulsive perturbations, Journal of Applied Mathematics 2012 (1) (2012) 1-19.

[13] L. S. Wang, R. Xu, A stage-structured predator-prey system with impulsive effect and holling type-II functional response, Journal of Mathematical Research with Applications 31 (1) (2011) $147-156$.

[14] D. He, W. Huang, Dynamics analysis of a population biological system with functional response and impulsive perturbations, Journal of Jilin University 46 (4) (2008) 607-612.

[15] X. Yu, S. Yuan, T. Zhang, Asymptotic properties of stochastic nutrient-plankton food chain models with nutrient recycling, Nonlinear Analysis Hybrid Systems 34 (2019) 209-225.

[16] X. Yu, S. Yuan, T. Zhang, Survival and ergodicity of a stochastic phytoplankton-zooplankton model with toxin-producing phytoplankton in an impulsive polluted environment, Applied Mathematics and Computation 347 (2019) 249-264.

[17] G. Zeng, L. Chen, L. Sun, Existence of periodic solution of order one of planar impulsive autonomous system, Journal of Computational and Applied Mathematics 186 (3) (2006) 466481.

[18] G. Jiang, Q. Lu, Impulsive state feedback control of a predator-prey model, Journal of Computational Applied Mathematics 200 (1) (2007) 193-207.

[19] L. Nie, Z. Teng, L. Hu, J. Peng, Qualitative analysis of a modified leslie-gower and hollingtype ii predator-prey model with state dependent impulsive effects, Nonlinear Analysis Real World Applications 11 (3) (2019) 1364-1373.

[20] L. Chen, K. Shimamoto, Emerging roles of molecular chaperones in plant innate immunity, Journal of General Plant Pathology 77 (1) (2011) 1-9.

[21] Z. Zheng, Y. Zhang, S. Jing, Nonlinear impulsive differential and integral inequalities with nonlocal jump conditions, Journal of Inequalities Applications 2018 (1) (2018) 170.

[22] P. Ghosh, J. F. Peters, Impulsive differential equation model in methanol poisoning detoxification, Journal of Mathematical Chemistry 58 (1) (2020) 126-145.

[23] Q. Liu, M. Zhang, L. Chen, State feedback impulsive therapy to sis model of animal infectious diseases, Physica A: Statistical Mechanics and its Applications 516 (2019) 222-232. 
[24] Y. Tian, S. Tang, R. A. Cheke, Nonlinear state-dependent feedback control of a pest-natural enemy system, Nonlinear Dynamics 94 (3) (2018) 2243-2263.

[25] M. U. Akhmet, On the general problem of stability for impulsive differential equations, Journal of Mathematical Analysis Applications 288 (1) (2018) 182-196.

[26] Y. Peng, Y. Li, T. Zhang, Global bifurcation in a toxin producing phytoplanktonczooplankton system with prey-taxis, Nonlinear Analysis Real World Applications 61 (6642) (2021) 103326.

[27] M. Huang, X. Song, Modeling and qualitative analysis of diabetes therapies with state feedback control, International Journal of Biomathematics 07 (4) (2014) 1450035.

[28] Z. Liang, G. Pang, X. Zeng, Y. Liang, Qualitative analysis of a predator-prey system with mutual interference and impulsive state feedback control, Nonlinear Dynamics 87 (3) (2016) 1-15.

[29] C. Liu, P. Liu, Complex dynamics in a harvested nutrient-phytoplankton-zooplankton model with seasonality, Mathematical Problems in Engineering 2014 (13) (2014) 565-565.

[30] A. Sharma, A. Sharma, K. Agnihotri, Dynamical analysis of a harvesting model of phytoplankton-zooplankton interaction, World Academy of Science, Engineering and Technology International Journal of Mathematical, Computational, Physical and Quantum Engineering 8 (1) (2014) 1007-1012.

[31] D. Li, Y. Liu, H. Cheng, Dynamic complexity of a phytoplankton-fish model with the impulsive feedback control by means of poincare map, Complexity 2020 (2020) 1-13.

[32] S. Tang, B. Tang, A. Wang, Y. Xiao, Holling II predator-prey impulsive semi-dynamic model with complex poincaré map, Nonlinear Dynamics 81 (3) (2015) 1575-1596.

[33] Y. Tian, K. Sun, L. Chen, Geometric approach to the stability analysis of the periodic solution in a semi-continuous dynamic system, International Journal of Biomathematics 7 (02) (2014) $157-163$.

[34] Y. Tian, S. Tang, R. A. Cheke, Nonlinear state-dependent feedback control of a pest-natural enemy system, Nonlinear Dynamics 94 (3) (2018) 2243-2263. 